\title{
Limitations of rapid myelin water quantification using 3D bSSFP
}

\author{
C. Lenz • M. Klarhöfer · K. Scheffler
}

Received: 12 February 2009 / Revised: 26 March 2010 / Accepted: 30 March 2010 / Published online: 28 April 2010 (C) ESMRMB 2010

\begin{abstract}
Object Imaging of the myelin water fraction (MWF) is conventionally performed using a multi-echo spin-echo sequence. This technique requires long acquisition times and therefore often suffers from a lack of volume coverage. In this work, the application of 3D balanced steady-state free precession (bSSFP) sequences to extract high-resolution myelin water maps is discussed.

Materials and Methods Based on a two-pool water exchange model, an approximate bSSFP signal equation is derived and applied to fit the flip angle dependence of the in vivo bSSFP signal. Thereby, the MWF and signal amplitude are fitted, while a priori assumptions are made for the other parameters of the two-pool system.

Results The effects of magnetization transfer, finite RF pulses, $B_{0}$ and $B_{1}$ inhomogeneities, as well as variation of the constant fit parameters, are investigated. Acquisition and calculation of quantitative, high-resolution MWF maps from white matter of healthy volunteers based on bSSFP is feasible and averaged MWF fit results agree with literature. However, results from numerical simulations indicate a severe dependence of the derived MWF values on the constant two-pool parameters.

Conclusion The demonstrated dependence of the MWF on the two-pool parameters considerably impairs the applicability of the proposed method.
\end{abstract}

C. Lenz $(\varangle) \cdot$ M. Klarhöfer $\cdot$ K. Scheffler

Division of Radiological Physics,

University of Basel Hospital, Petersgraben 4,

4031 Basel, Switzerland

e-mail: claudia.lenz@unibas.ch
Keywords Myelin water fraction - Balanced SSFP . Quantitative imaging · Brain microstructure

\section{Introduction}

Myelin is an electrically insulating material that consists of multiple lipid bilayers surrounding the axons of neurons and mainly appears in white matter (WM) and to a small amount in gray matter (GM) of the peripheral and central nervous system of vertebrates. Its central function is to increase the velocity of the electrical signal conducted along the axons. The myelin sheath tightly encloses water, which exhibits therefore special magnetic resonance properties. Compared to the intra- and extracellular water of the axon, the myelin water experiences a shorter transverse relaxation $T_{2}$. Based on multicompartment analysis of this $T_{2}$ decay, quantification of the myelin water fraction (MWF) becomes feasible and provides insight into pathologies of WM diseases such as multiple sclerosis (MS).

The existence of the short $T_{2}$ of myelin water is known now for 30 years and was first shown in in vitro studies [1-3]. The most important method for in vivo pixelwise mapping of the MWF was introduced by MacKay et al. [4]. In their work, a single-slice 32-echo spin-echo sequence was used to acquire the multicompartment $T_{2}$ decay. The data was analyzed with the help of a non-negative least-squares algorithm $[5,6]$, and the MWF was calculated according to the size of the short $T_{2}$ compartment. This technique was later used for the investigation into demyelinating processes in the course of MS, where reduced MWF in lesions was observed [7-9]. Recently, the procedure of MacKay et al. was successfully applied in determining the MWF of patients suffering from other diseases like schizophrenia [10] or phenylketonuria [11]. Nevertheless, this approach has the disadvantages of 
long acquisition times (about 25 min for one slice) and a lack of volume coverage due to the single-slice measurement technique. So far, myelin water imaging with whole-brain coverage is a relatively new field with very little existing published work [12-14].

Besides the existence of the multi-component $T_{2}$ of WM and $\mathrm{GM}$, there is evidence that the longitudinal relaxation $T_{1}$ is as well composed of more than one component and that $T_{1}$ of myelin water is specifically shorter than $T_{1}$ of the intraand extracellular water pools of the axon [14-17]. However, direct observation of multiple $T_{1}$ components is hindered due to the short timescale of exchange between the water compartments. Combined $T_{2}$ and $T_{1}$ measurements or application of a sequence with combined contrast were shown to be feasible solutions for the extraction of multi-component $T_{1}$ relaxation $[14,15,17]$.

Based on the idea that balanced steady-state free precession (bSSFP) sequences [18] can successfully be used for the quantitative assessment of magnetization transfer (MT) [19], this technique was applied for the quantification of myelin water. Imaging with bSSFP benefits from very short acquisition times (about $2 \mathrm{~min}$ for one 3D data set), high resolution and excellent signal-to-noise ratio (SNR) [20]. The signal formation in bSSFP is usually described by the Freeman-Hill formula [21,22]. However, in most biological tissues like WM or GM, the signal is more completely described by a complex composition of relaxation instead of the classical single-pool behavior. In this work, a complete mathematical derivation of an extended bSSFP signal equation, based on a two-pool water model describing WM and GM, is presented. The model consists of a short pool (myelin water, abbreviation s), a long pool (extracellular and axonal water, abbreviation 1) and additionally includes proton exchange between the pools. The extended bSSFP signal equation is a function of the repetition time TR, the echo time TE, the flip angle $\alpha$, the relaxation times of the two pools $\left(T_{2}^{s}, T_{2}^{l}, T_{1}^{s}, T_{1}^{l}\right)$, the exchange rate $k$ and most important the MWF.

The extended bSSFP signal equation is validated by numerical simulations of the full set of non-simplified ordinary differential equations (ODE). Moreover, the influence of finite radiofrequency (RF) pulses and MT, as well as the dependence on $B_{0}$ and $B_{1}$ inhomogeneities is carefully investigated in this work. The new signal equation is used to fit data from in vivo 3D bSSFP measurements with varying flip angles. The MWF and the signal amplitude are fitted as single unknowns, while keeping the remaining parameters of the two-pool system (relaxation times of the two pools and exchange rate) constant. Both region of interest (ROI) and pixelwise results from the fitting of axial, sagittal and coronal views of healthy subjects are presented. The effect of variation of the relaxation times and the exchange rate on the MWF fit is discussed.

\section{Materials and methods}

Theoretical background

\section{Conventional bSSFP signal equation}

In the case of a single water compartment, the steady state of bSSFP is derived from the Bloch equations using partial integration, resulting in the well-known Freeman-Hill formula [21,22]:

$S=M_{0} \sin \alpha \frac{\left(1-E_{1}\right) \sqrt{E_{2}}}{1-E_{1} E_{2}-\left(E_{1}-E_{2}\right) \cos \alpha}$.

This equation is valid for centered echoes $(\mathrm{TE}=\mathrm{TR} / 2)$ and alternating excitations $( \pm \alpha)$. Furthermore, $E_{1,2}=$ $\exp \left(-\mathrm{TR} / T_{1,2}\right)$ and $M_{0}$ is the equilibrium magnetization. For WM and GM, which are complex biological tissues, the bSSFP signal is more completely described by a complex composition of relaxation instead of the classical single-pool behavior. In the following, a complete mathematical derivation of an extended bSSFP signal equation will be presented, in order to characterize the bSSFP behavior of the different water pools in brain tissue. For the mathematical derivation, a similar nomenclature as in references [14] and [23] is used.

\section{Two-pool water model}

To accurately describe the bSSFP signal of WM and GM, a theoretical two-pool model is used. The short pool consists of myelin water, whereas the extracellular and the axonal water are combined to the long pool. The relaxation times $T_{1}^{s}$ and $T_{2}^{s}$ belong to the short pool, $T_{1}^{l}$ and $T_{2}^{l}$ to the long one (Fig. 1). Furthermore, the two components undergo a proton exchange that is indicated by the exchange rates $k_{s l}$ and $k_{l s}$. Exchange is specified by water molecules diffusing through ion channels of the myelin bilayer either from the short to the long pool $\left(k_{s l}\right)$ or vice versa $\left(k_{l s}\right)$. The exchange rates are the reciprocals of the mean residence times $\tau_{s l}$ and $\tau_{l s}$, the average time a proton remains in one pool before diffusing to the other. In kinetic equilibrium, the two exchange rates are related through the fractional pool sizes by $k_{s l}=(1 / M W F-1) k_{l s}$. The MWF stands for the molar fraction of the short pool and is defined as $M W F=M_{0}^{s} /\left(M_{0}^{s}+M_{0}^{l}\right)$, where $M_{0}^{s}\left(M_{0}^{l}\right)$ denotes the equilibrium magnetization of the short (long) pool. Aside from that, a general exchange rate can be defined as $k=k_{s l}+k_{l s}$, whereby $k_{s l}=(1-M W F) \cdot k$ and $k_{l s}=M W F \cdot k$ become valid. The timescale of exchange $\tau$ is believed to be long on a $T_{2}$ timescale $(\tau>100 \mathrm{~ms})$, because two $T_{2}$ components are observed. On the other hand, $\tau$ needs to be short on a $T_{1}$ timescale $\left(\tau<700 \mathrm{~ms}\right.$ ), because in conventional $T_{1}$ measurements only one component is observed, despite the existence of two physically different $T_{1}$. The literature value 


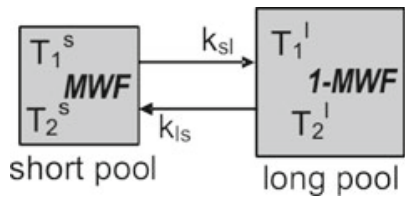

Fig. 1 Physical properties of the two water pools describing WM and GM. The short pool has relaxation times $T_{1}^{s}, T_{2}^{s}$ and the fractional pool size MWF, whereas $T_{1}^{l}, T_{2}^{l}$ and (1-MWF) belong to the long pool. Proton exchange is described by the two exchange rates $k_{s l}$ and $k_{l s}$

for $\tau$ is $\sim 200 \mathrm{~ms}[17,24,25]$, which lies between the two limits and corresponds to $k \sim 5 \mathrm{~s}^{-1}$.

\section{Modified Bloch equations}

The magnetization of the short pool will decrease by the factor $k_{s l} \mathbf{M}^{s}$ and increase by $k_{l s} \mathbf{M}^{l}$, if exchange between the two pools occurs. On the other hand, the magnetization of the long pool will rise by $k_{l s} \mathbf{M}^{l}$ and decrease by $k_{s l} \mathbf{M}^{s}$. This leads to modified 6-D Bloch equations [26], which describe all spatial components of the two-pool magnetization vector $\mathbf{M}=\left[M_{x}^{s}, M_{x}^{l}, M_{y}^{s}, M_{y}^{l}, M_{z}^{s}, M_{z}^{l}\right]$ separately:

$$
\begin{aligned}
& \frac{d M_{x}^{s}}{d t}=\omega_{0} M_{y}^{s}-\frac{M_{x}^{s}}{T_{2}^{s}}-k_{s l} M_{x}^{s}+k_{l s} M_{x}^{l} \\
& \frac{d M_{x}^{l}}{d t}=\omega_{0} M_{y}^{l}-\frac{M_{x}^{l}}{T_{2}^{l}}-k_{l s} M_{x}^{l}+k_{s l} M_{x}^{s} \\
& \frac{d M_{y}^{s}}{d t}=-\omega_{0} M_{x}^{s}-\frac{M_{y}^{s}}{T_{2}^{s}}-k_{s l} M_{y}^{s}+k_{l s} M_{y}^{l}+\omega_{1}(t) M_{z}^{s},
\end{aligned}
$$$$
\frac{d M_{y}^{l}}{d t}=-\omega_{0} M_{x}^{l}-\frac{M_{y}^{l}}{T_{2}^{l}}-k_{l s} M_{y}^{l}+k_{s l} M_{y}^{s}+\omega_{1}(t) M_{z}^{l},
$$

$\frac{d M_{z}^{s}}{d t}=\frac{M_{0}^{s}-M_{z}^{s}}{T_{1}^{s}}-k_{s l} M_{z}^{s}+k_{l s} M_{z}^{l}-\omega_{1}(t) M_{y}^{s}$,

$\frac{d M_{z}^{l}}{d t}=\frac{M_{0}^{l}-M_{z}^{l}}{T_{1}^{l}}-k_{l s} M_{z}^{l}+k_{s l} M_{z}^{s}-\omega_{1}(t) M_{y}^{l}$.

Here, $M_{0}^{s}\left(M_{0}^{l}\right)$ is again the abbreviation for the equilibrium magnetization of the short (long) pool, $\omega_{0}=\gamma\left|\boldsymbol{B}_{0}\right|$ and $\omega_{1}(t)=\gamma\left|\boldsymbol{B}_{1}(t)\right|$. Moreover, it is assumed that $\omega_{0}=\omega_{0}^{s}=$ $\omega_{0}^{l}$. The modified Bloch equations describe the cases of excitation, relaxation and exchange.

\section{Solutions to the modified Bloch equations}

Although, in principle it may be possible to solve the system of differential equations (Eq. 2a-2f), the solution is complex and cumbersome. Therefore, a different procedure is suggested, which closely follows the vector model description introduced by Carr [27] to derive the signal behavior of bSSFP. To separate excitation and relaxation, instanta- neously acting RF pulses are assumed. Using the matrix notation developed by Jaynes [28], the solution for excitation is written as a rotation around the $\mathrm{x}$-axis (without loss of generality):

$\mathbf{M}_{n}^{+}=\mathbf{R}_{x}(\alpha) \mathbf{M}_{n}^{-}=\left[\begin{array}{cccccc}1 & 0 & 0 & 0 & 0 & 0 \\ 0 & 1 & 0 & 0 & 0 & 0 \\ 0 & 0 & \cos \alpha & 0 & \sin \alpha & 0 \\ 0 & 0 & 0 & \cos \alpha & 0 & \sin \alpha \\ 0 & 0 & -\sin \alpha & 0 & \cos \alpha & 0 \\ 0 & 0 & 0 & -\sin \alpha & 0 & \cos \alpha\end{array}\right] \mathbf{M}_{n}^{-}$,

where $\alpha$ is the flip angle, and $\mathbf{M}_{n}^{-}$is the 6-D magnetization vector before the $n$th RF pulse. The solution for relaxation is found analogously to the 3 -D classical case $[21,22]$. The resulting relaxation matrix is given by:

$\mathbf{E}(t)=\left[\begin{array}{cccccc}E_{2}^{s} & 0 & 0 & 0 & 0 & 0 \\ 0 & E_{2}^{l} & 0 & 0 & 0 & 0 \\ 0 & 0 & E_{2}^{s} & 0 & 0 & 0 \\ 0 & 0 & 0 & E_{2}^{l} & 0 & 0 \\ 0 & 0 & 0 & 0 & E_{1}^{s} & 0 \\ 0 & 0 & 0 & 0 & 0 & E_{1}^{l}\end{array}\right]$

where $E_{1,2}^{s}=\exp \left(-t / T_{1,2}^{s}\right), E_{1,2}^{l}=\exp \left(-t / T_{1,2}^{l}\right)$.

To solve the modified Bloch equations for exchange, it is additionally assumed that exchange processes decouple from relaxation processes within the short repetition times usually applied in bSSFP imaging. This approach is valid, as long as fractional pool size modifications from longitudinal relaxation processes are negligible within TR. This corresponds to an instantaneous appearance of exchange, similar to the concept of instantaneous rotation allowing the decoupling of excitation and relaxation. The part of the modified Bloch equations containing only exchange parameters reads:

$\frac{d \mathbf{M}}{d t}=\left[\begin{array}{cccccc}-k_{s l} & k_{l s} & 0 & 0 & 0 & 0 \\ k_{s l} & -k_{l s} & 0 & 0 & 0 & 0 \\ 0 & 0 & -k_{s l} & k_{l s} & 0 & 0 \\ 0 & 0 & k_{s l} & -k_{l s} & 0 & 0 \\ 0 & 0 & 0 & 0 & -k_{s l} & k_{l s} \\ 0 & 0 & 0 & 0 & k_{s l} & -k_{l s}\end{array}\right] \mathbf{M}$

This differential equation has the general solution $\mathbf{M}(t)=$ $\mathbf{A}(t) \cdot \mathbf{M}_{0}$, where $\mathbf{A}(\mathrm{t})$ is the exchange matrix:

$\mathbf{A}(t)=\left[\begin{array}{llllll}a & b & 0 & 0 & 0 & 0 \\ c & d & 0 & 0 & 0 & 0 \\ 0 & 0 & a & b & 0 & 0 \\ 0 & 0 & c & d & 0 & 0 \\ 0 & 0 & 0 & 0 & a & b \\ 0 & 0 & 0 & 0 & c & d\end{array}\right]$

with

$a=-(M W F-1) e^{-k t}+M W F, \quad b=-M W F\left(e^{-k t}-1\right)$

$c=(M W F-1)\left(e^{-k t}-1\right), \quad d=M W F\left(e^{-k t}-1\right)+1$. 
$k_{S l}$ and $k_{l s}$ were replaced by the corresponding expressions including $k$ and MWF. Although the concatenation of relaxation and exchange is not a general solution for longer TR, it can be applied in the limit of short TR. The limit of TR $\rightarrow$ 0 leads to the case of commuting relaxation and exchange matrices: $[\mathrm{E}(\mathrm{TR} \rightarrow 0), \mathrm{A}(\mathrm{TR} \rightarrow 0)] \rightarrow 0$.

\section{Extended bSSFP signal equation}

The extended steady-state equation can now be derived according to standard procedures [29] with the solutions for excitation, relaxation and exchange from above. RF phase alternations are described by $\mathbf{R}_{\mathrm{z}}$ :

$\mathbf{R}_{z}\left(\Phi=180^{\circ}\right)=\left[\begin{array}{cccccc}-1 & 0 & 0 & 0 & 0 & 0 \\ 0 & -1 & 0 & 0 & 0 & 0 \\ 0 & 0 & -1 & 0 & 0 & 0 \\ 0 & 0 & 0 & -1 & 0 & 0 \\ 0 & 0 & 0 & 0 & 1 & 0 \\ 0 & 0 & 0 & 0 & 0 & 1\end{array}\right]$.

For bSSFP, the magnetization directly after the $n$th RF-pulse yields $\mathbf{M}_{n}^{+}=\mathbf{R}_{\mathrm{z}} \mathbf{R}_{\mathrm{x}}(\alpha) \mathbf{M}_{n}^{-}$, where $\mathbf{M}_{n}^{-}$is the magnetization before the $n$th RF-pulse. The magnetization before the $(n+1)$ th pulse yields (assuming relaxation takes place before exchange):

$\mathbf{M}_{n+1}^{-}=\mathbf{A}(T R)\left[\mathbf{E}(T R) \mathbf{M}_{n}^{+}+\mathbf{M}_{0}\right]$,

with the equilibrium magnetization given by:

$\mathbf{M}_{0}=\left[\begin{array}{c}0 \\ 0 \\ 0 \\ 0 \\ M_{0}^{s}\left(1-E_{1}^{s}\right) \\ M_{0}^{l}\left(1-E_{1}^{l}\right)\end{array}\right]$.

In steady state, the eigenvalue equation takes the form $\mathbf{M}_{n}^{-}=$ $\mathbf{M}_{n+1}^{-}$, and the final result becomes (for centered echoes with $\mathrm{TE}=\mathrm{TR} / 2$ and the case relaxation before exchange):

$\mathbf{M}_{n}^{+}(\infty)=\sqrt{\mathbf{E}} \mathbf{R}_{x}\left[\mathbf{I}_{6}-\mathbf{A} \mathbf{E} \mathbf{R}_{z} \mathbf{R}_{x}\right]^{-1} \mathbf{A} \mathbf{M}_{0}$,

where all matrices are evaluated at time point TR. The total signal amplitude is calculated according to: $S=|\mathbf{M}|_{x y}^{s}+$ $|\mathbf{M}|_{x y}^{l}$. The analytical solution yields the final extended bSSFP signal equation:

$S \propto \sin \alpha \frac{A+B \cos \alpha}{C+D \cos \alpha+E \cos 2 \alpha}$,

with abbreviations

$A, B, C, D, E=f\left(T R, E_{1,2}^{s, l}, k, M W F\right)$.

A list of the full expressions is given in section "Appendix". The limit where only the short pool exists with fraction
$\mathrm{MWF}=100 \%$ and zero exchange leads to the well-known standard bSSFP equation (Eq. 1). Equations 10 and 11 represent the case where relaxation before exchange occurs. Comparison to the case of exchange before relaxation showed negligible signal differences. Therefore, only relaxation before exchange will be considered below.

\section{Numerical simulations}

All numerical simulations and data analysis were performed using Matlab 2007b (The MathWorks, Inc., Natick, MA, United States).

To verify Eq. 11 and to justify the separation of relaxation and exchange processes, numerical simulations of the full set of non-simplified ordinary differential equations (ODE) (see Eq. 2a-2f) were conducted. A standard ODE solver was used to simulate the signal as a function of RF pulse characteristics (flip angle $\alpha, \mathrm{RF}$ pulse duration $T_{\mathrm{RF}}$ ), sequence characteristics (repetition time TR) and two-pool properties (relaxation times, MWF and exchange rate $k$ ). Rectangular shaped RF pulses were used for excitation.

To investigate the effects of MT, the modified Bloch equations were extended to $9 \mathrm{D}$ to comprise an additional restricted pool consisting of membranes and macromolecules. Numerical simulations of the 9-D ODE set were performed taking into account saturation of the restricted pool protons, as well as proton exchange with the two free water pools. In general, saturation of the restricted pool protons is achieved by off-resonance irradiation affecting the longitudinal magnetization of the restricted pool and is described by the mean saturation rate [19]:

$$
\langle W(\Delta)\rangle=\frac{\pi}{T_{R F}} \int_{0}^{T_{R F}} \omega_{1}^{2}(t) d t G(\Delta),
$$

where $\Delta$ is the frequency offset and $G(\Delta)$ the absorption line shape. For the simulations (and measurements) of this work, on-resonance RF pulses were applied, which results in $\Delta \rightarrow 0$. The mean saturation rate $W(\Delta \rightarrow 0)$ is calculated as a function of the flip angle $\alpha$ and the RF pulse duration $\mathrm{T}_{\mathrm{RF}}$ according to Eq. (12) and based on a Super-Lorentzian line shape, yielding $G(0)=1.4 \times 10^{-5} \mathrm{~s}^{-1}$ for a $T_{2}$ of the restricted pool of $12 \mu \mathrm{s}$ [30].

\section{In vivo experiments}

Measurements were conducted on a Siemens Avanto 1.5T system (Siemens Healthcare, Erlangen, Germany) in 3D with sagittal orientation based on a $144 \times 192 \times 192$ matrix with $1.3 \mathrm{~mm}$ isotropic resolution. The protocol for quantitative MWF estimation consisted of: 
1. A carefully conducted second-order manual shim for correction of main magnetic field inhomogeneities resulting in frequency variations of less than $15 \mathrm{~Hz}$ within the shimmed volume containing the whole brain.

2. An MPRAGE sequence for anatomical reference and segmentation of WM $(\mathrm{TR} / \mathrm{TE}=1760 / 4.61 \mathrm{~ms}$, inversion time $\mathrm{TI}=906 \mathrm{~ms}$, flip angle $\alpha=7^{\circ}$, bandwidth $=$ $130 \mathrm{~Hz} /$ pixel).

3. Multiple bSSFP sequences with 8 varying flip angles $\alpha=$ $4,8,12, \ldots, 32^{\circ}$ and non-selective rectangular RF pulses with $T_{\mathrm{RF}}=2 \mathrm{~ms}$ to minimize MT effects (see Fig. $3 \mathrm{~b}$ and [31] $)(\mathrm{TR} / \mathrm{TE}=5.4 / 2.7 \mathrm{~ms}$, bandwidth $=500 \mathrm{~Hz} / \mathrm{pixel})$.

4. A multi-slice $B_{1}$ field map sequence ( 20 slices, $5 \mathrm{~mm}$ slice thickness, $64 \times 64$ matrix, $4 \mathrm{~mm}$ in plane resolution) for the assessment of flip angle deviations based on stimulated echoes resulting from a multi-pulse sequence $(\alpha-\alpha-\alpha)$, analogous to the scheme described in [32].

An acquisition time of $25 \mathrm{~min}$ is required to measure the whole protocol (MPRAGE: $3 \mathrm{~min}$, bSSFP: $8 \times 2.5 \mathrm{~min}, B_{1}$ field map: $2 \mathrm{~min}$ ). In total, six healthy volunteers were investigated. Informed consent was obtained from all participants, and experiments were approved by local ethics committee.

Postprocessing and data analysis

For image registration and brain extraction, the software packages FSL [33] and AFNI [34] were used. The effective measured $B_{1}$ field is depicted as percentage difference from the actual flip angle. According to that, flip angle deviations were calculated pixelwise after $B_{1}$ image registration and data interpolation performed with FSL.

The correct assessment of myelin water demands the elimination of possible MT contributions. Therefore, a long RF pulse duration of $2 \mathrm{~ms}$ was chosen for the experimental setup of the bSSFP sequence [31]. However, the derivation of the new bSSFP signal equation was based on instantaneously acting RF pulses. Recently, Bieri and Scheffler showed that long pulse durations in bSSFP imaging may lead to a divergence between bSSFP signal prediction and measurement data, making a finite RF pulse correction necessary [35]. They suggest the following modification to the transverse relaxation $T_{2}$ (for non-selective rectangular RF-pulses):

$$
\begin{aligned}
T_{2 \text { corr }} & =\left(1-\zeta \frac{T_{R F}}{T R}\right)^{-1} T_{2} \text { with } \\
\zeta & =0.68-0.125\left(1+\frac{T_{R F}}{T R}\right) \frac{T_{2}}{T_{1}},
\end{aligned}
$$

where $T_{\mathrm{RF}}$ is the duration of the RF pulse. This correction was consistently included in the derived bSSFP signal equation.

One axial, one sagittal and one coronal section per volunteer were selected for analysis. The MWF was fitted using the finite RF pulse corrected bSSFP signal equation (Eq. 11) as fit equation and a nonlinear least squares (NLLS) approach as fit procedure. The fit was performed as a function of the 8 different $\alpha$. The distance between acquired data and model was minimized with respect to the MWF and the signal amplitude. The parameters $T_{1}^{s}, T_{1}^{l}, T_{2}^{s}, T_{2}^{l}$ and $k$ were kept constant, whereas TR is known from the measurements $(5.4 \mathrm{~ms})$. For the fit of WM regions, a priori values were chosen according to WM literature values: $T_{2}^{s}=10 \mathrm{~ms}, T_{2}^{l}=80 \mathrm{~ms}, T_{1}^{s}=$ $400 \mathrm{~ms}, T_{1}^{l}=900 \mathrm{~ms}$ and $k=5 \mathrm{~s}^{-1}[4,17,24,25,36]$. The constant parameters for GM region fitting were fixed analogously according to GM literature values: $T_{2}^{s}=12 \mathrm{~ms}, T_{2}^{l}=$ $90 \mathrm{~ms}, T_{1}^{s}=600 \mathrm{~ms}, T_{1}^{l}=1,400 \mathrm{~ms}$ and $k=5 \mathrm{~s}^{-1}$.

For the pixelwise fits, a Wiener filter with kernel size 3 was first employed on all images to increase SNR without substantial loss of image details. WM pixels were then segmented based on signal thresholds of the MPRAGE images. The fit of the MWF solely was applied to the segmented WM pixels, thereby using the constant WM parameters given above. Only MWF fit results with standard errors $<100 \%$ were allowed.

\section{Results}

Numerical simulations

Figure 2 depicts a comparison of the derived bSSFP signal equation (Eq. 11) with the numerical simulation of the

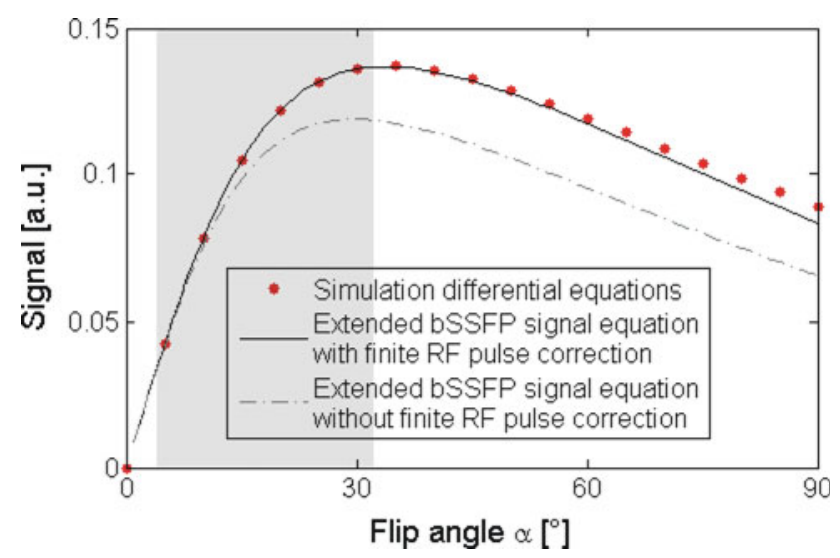

Fig. 2 Comparison of the numerical simulation of the differential Eq. ((2a)-(2f)) with the derived extended bSSFP equation using WM parameters: $T_{2}^{s}=10 \mathrm{~ms}, T_{2}^{l}=80 \mathrm{~ms}, T_{1}^{s}=400 \mathrm{~ms}, T_{1}^{l}=$ $900 \mathrm{~ms}, k=5 \mathrm{~s}^{-1}, \mathrm{MWF}=20 \%, T_{\mathrm{RF}}=2 \mathrm{~ms}, \mathrm{TR}=2 \cdot \mathrm{TE}=5.4 \mathrm{~ms}$. The gray dashed line shows the plot of the derived bSSFP signal equation without finite RF pulse correction, the black line illustrates the bSSFP equation including the correction. The total signal is depicted as a function of the flip angle $\alpha$, the shaded gray area displays the measurement range. Excellent correspondence between the simulation of the differential equations and the finite RF pulse corrected bSSFP signal equation is found in the range of measurements, therefore justifying the application of the finite RF pulse correction 

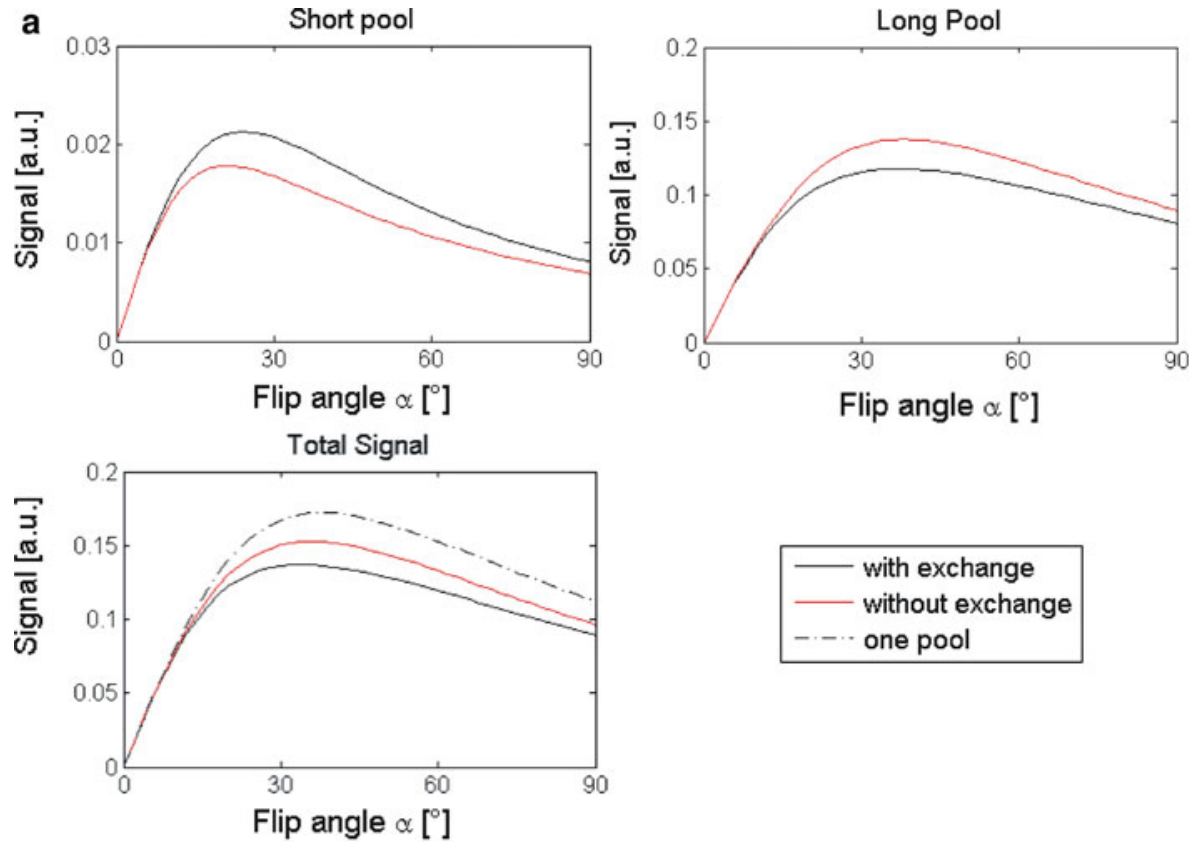

Flip angle $\alpha\left[{ }^{\circ}\right]$
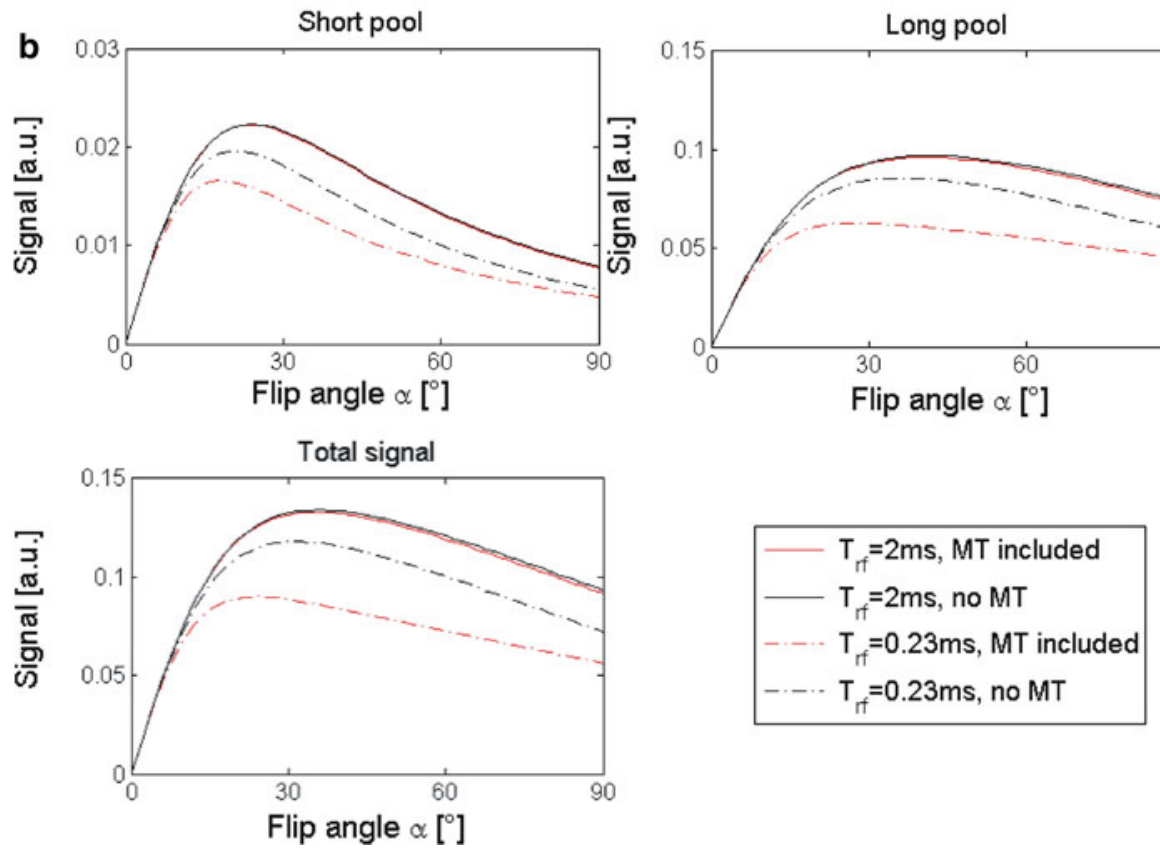

Fig. 3 a Numerical WM bSSFP simulation of the two-pool Bloch equations showing the signal of the short pool, the signal of the long pool and the total signal as a function of the flip angle. Black line: simulation with exchange $\left(k=5 \mathrm{~s}^{-1}, \mathrm{MWF}=20 \%\right)$, red line: simulation without exchange $\left(k=0 \mathrm{~s}^{-1}, \mathrm{MWF}=20 \%\right)$ and broken gray line: simulation of one existing pool $\left(k=0 \mathrm{~s}^{-1}, \mathrm{MWF}=100 \%\right)$. Other parameters used were: $T_{2}^{s}=10 \mathrm{~ms}, T_{2}^{l}=80 \mathrm{~ms}, T_{1}^{s}=400 \mathrm{~ms}, T_{1}^{l}=900 \mathrm{~ms}, T_{\mathrm{RF}}=$ $2 \mathrm{~ms}$ and $\mathrm{TR}=2 \cdot \mathrm{TE}=5.4 \mathrm{~ms}$. b Numerical WM bSSFP simulation of the 9-D Bloch equations including an additional third restricted pool (abbreviation $r$ ) to investigate the connection between MT and RF-pulse

full set of non-simplified ODE (Eq. 2a-2f). Within the range of experimentally applied flip angles $\left(4^{\circ}-32^{\circ}\right)$, the extended bSSFP steady-state solution clearly underestimates duration. The signal of the short pool, the signal of the long pool and the total signal are illustrated as a function of the flip angle. Parameters used were: $T_{2}^{s}=10 \mathrm{~ms}, T_{2}^{l}=80 \mathrm{~ms}, T_{1}^{s}=400 \mathrm{~ms}, T_{1}^{l}=900 \mathrm{~ms}, \mathrm{MWF}=$ $20 \%, T_{1}^{r}=1,000 \mathrm{~ms}, T_{2}^{r}=12 \mu \mathrm{s}, \mathrm{TR}=2$. TE $=5.4 \mathrm{~ms}, k_{s l}$ $=4 \mathrm{~s}^{-1}, k_{l s}=1 \mathrm{~s}^{-1}, k_{r s}=30 \cdot 0.2 \mathrm{~s}^{-1}, k_{s r}=4.5 \cdot 0.2 \mathrm{~s}^{-1}, k_{r l}=$ $30 \cdot 0.8 \mathrm{~s}^{-1}$ and $k_{l r}=4.5 \cdot 0.8 \mathrm{~s}^{-1}$. Moreover: fraction of the restricted pool $F_{r}=15 \%$, therefore MWF $+(1-\mathrm{MWF}) \equiv 85 \%$ of the total size of all pools. The signals are plotted with and without MT, based on two $\mathrm{RF}$ pulse durations of $2 \mathrm{~ms}$ and $230 \mu \mathrm{s}$

the simulated values by $15 \%$ at the maximum difference. This is due to the assumed instantaneously acting RF pulses, which is in contradiction to the long RF pulse duration chosen 

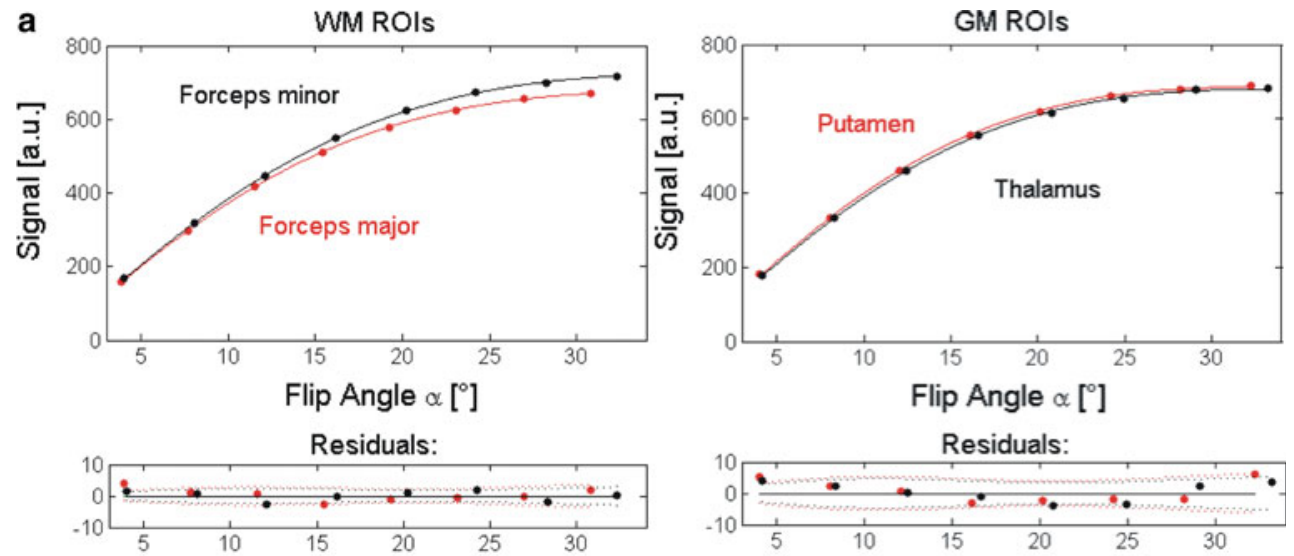

Flip Angle $\alpha\left[{ }^{\circ}\right]$

b

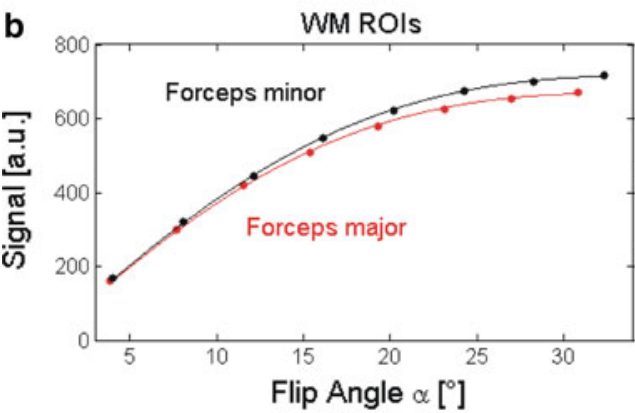

Residuals:

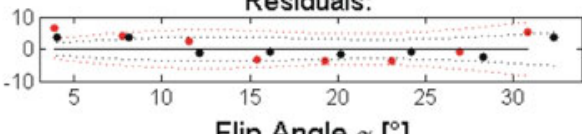

Flip Angle $\alpha\left[^{\circ}\right]$

Fig. 4 a Introductory examples to the two-pool bSSFP analysis showing MWF parameter fitting of two WM and two GM ROIs from one healthy subject. NLLS fit procedure and application of the finite RF pulse corrected extended bSSFP signal equation (Eq. 11) with either constant WM parameters $T_{2}^{s}=10 \mathrm{~ms}, T_{2}^{l}=80 \mathrm{~ms}, T_{1}^{s}=$ $400 \mathrm{~ms}, T_{1}^{l}=900 \mathrm{~ms}$ and $k=5 \mathrm{~s}^{-1}$ or constant GM parameters $T_{2}^{s}=12 \mathrm{~ms}, T_{2}^{l}=90 \mathrm{~ms}, T_{1}^{s}=600 \mathrm{~ms}, T_{1}^{l}=1,400 \mathrm{~ms}$ and $k=5 \mathrm{~s}^{-1}$

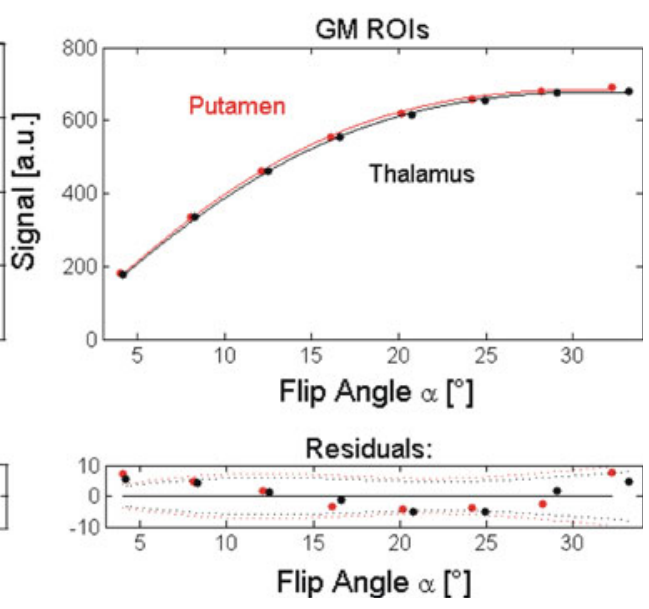

yield MWF results displayed in Table 1. b Single-pool fitting examples of the regions from Fig. 4a. NLLS fit procedure and application of the finite RF pulse corrected Freeman-Hill formula with either the constant WM parameter $T_{1}=850 \mathrm{~ms}$ or the constant GM parameter $T_{1}=1,200 \mathrm{~ms}$ lead to single-pool $T_{2}$ fit results given in Table 1 . Moreover, fitting residuals and $95 \%$ confidence intervals are presented. Sum of squares of the fitting residuals are given in Table 1

Table 1 ROI results derived from two-pool bSSFP model fitting and single pool fitting using the Freeman-Hill formula (ROI results \pm standard error)

\begin{tabular}{llll} 
WM & & GM & \\
\cline { 4 - 4 } Forceps minor & Forceps major & Putamen & Thalamus \\
\hline
\end{tabular}

Two-pool bSSFP fitting (Eq. 11)

\begin{tabular}{lllll} 
MWF [\%] & $15.4 \pm 1.5$ & $11.1 \pm 1.0$ & $5.8 \pm 2.0$ & $3.9 \pm 1.6$ \\
SSQ $^{\text {b }}$ & 29.7 & 18.6 & 92.9 & 68.1 \\
Single pool fitting (Eq. 1) & & & & \\
$T_{2}[\mathrm{~ms}]$ & $53.6 \pm 3.2$ & $58.7 \pm 2.0$ & $67.7 \pm 4.2$ & $70.7 \pm 3.6$ \\
$\mathrm{SSQ}^{\mathrm{b}}$ & 130.5 & 51.3 & 187.8 & 128.5 \\
\hline
\end{tabular}

${ }^{\text {a}}$ Fixed WM parameters: $T_{2}^{s}=10, T_{2}^{l}=80, T_{1}^{s}=400, T_{1}^{l}=900 \mathrm{~ms}, k=5 \mathrm{~s}^{-1}$; fixed GM parameters: $T_{2}^{s}=12, T_{2}^{l}=90, T_{1}^{s}=600, T_{1}^{l}=$ $1,400 \mathrm{~ms}, k=5 \mathrm{~s}^{-1}$

${ }^{\mathrm{b}}$ Sum of squares of the fit residuals

${ }^{c}$ Fixed WM parameter: $T_{1}=850 \mathrm{~ms}$; fixed GM parameter: $T_{1}=1,200 \mathrm{~ms}$ 


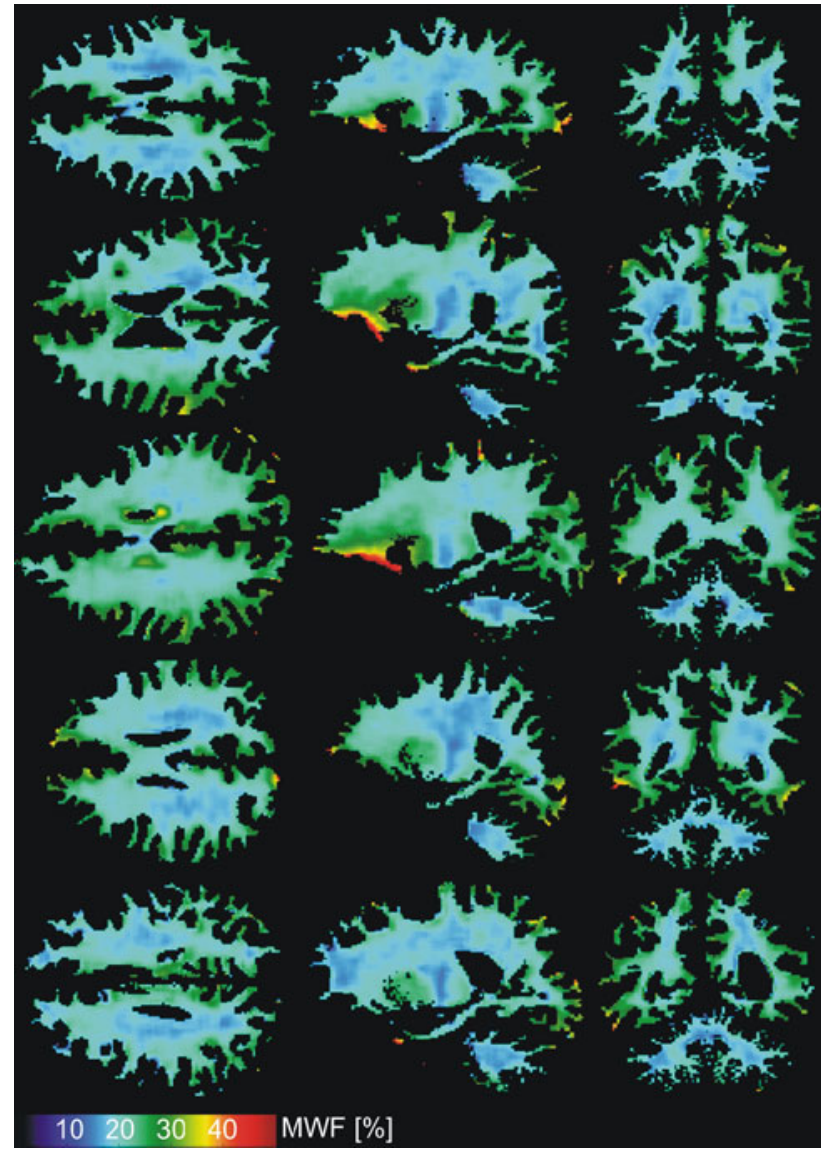

Fig. 5 Axial, sagittal and coronal sample images from five healthy volunteers showing $1.3 \mathrm{~mm}$ isotropic high-resolution MWF parameter estimates as derived from two-pool bSSFP model fitting using the NLLS algorithm and the extended bSSFP fit equation (Eq. 11). Analysis was solely applied to segmented WM pixels using the constant WM parameters $T_{2}^{s}=10 \mathrm{~ms}, T_{2}^{l}=80 \mathrm{~ms}, T_{1}^{s}=400 \mathrm{~ms}, T_{1}^{l}=900 \mathrm{~ms}$ and $k=5 \mathrm{~s}^{-1}$. Mean values with corresponding standard deviations are listed in Table 2

for experiments to exclude MT effects. The application of a finite RF pulse correction therefore becomes essential. Using the procedure proposed in [35] for the correction of transverse relaxation, finally good agreement $(<0.1 \%$ deviation at $\alpha=32^{\circ}$ ) between the analytical description of the two-pool bSSFP signal and the numerical simulation of the differential equations is achieved. In summary, provided that the finite RF pulse correction is included, the use of the derived twopool bSSFP signal equation is valid within the measurement range.

Figure 3a illustrates further results from the numerical simulations of the two-pool Bloch equations (Eq. 2a-2f). The signal amplitudes are shown for both pools and for the total signal separately. If exchange processes are taken into account, the short pool will gain signal intensity, which will in return be lost by the long pool. Due to the fast decay of the transverse magnetization of the short pool, less magne- tization is available for transfer to the long pool in comparison with the amount exchanged by the long component. The right plot depicts an important difference between the total signal including exchange and the one without exchange: the neglect of proton exchange would lead to an overestimation of the fitted MWF of about $10 \%$. In consequence, incorporating proton exchange is necessary to extract the correct MWF. Besides that, an obvious difference between the total signal for two pools and the signal for only one existing pool is observed.

Figure $3 b$ displays the results from the numerical simulations based on the Bloch equations comprising three pools: the two water pools described above and an additional restricted pool consisting of macromolecules and membranes. The simulations included saturation of the restricted pool, as well as proton exchange between all pools. Two RF pulse durations were investigated: $230 \mu$ s (a typical pulse duration used for MT experiments [31]) and $2 \mathrm{~ms}$, as it was applied in our measurements. For $T_{\mathrm{RF}}=230 \mu \mathrm{s}$, an evident signal difference between MT and no MT is observed, which represents the background of measuring MT effects. On the other hand, the correct assessment of the MWF demands the elimination of possible MT contributions. This can be achieved by choosing an RF pulse duration of $2 \mathrm{~ms}$. Due to the decrease in RF pulse bandwidth, the signal difference between MT and no MT becomes negligible small for $2 \mathrm{~ms}$ duration. Therefore, MT effects can definitely be excluded from the experiments performed in this study.

\section{In vivo experiments}

Quantitative MWF evaluation is exemplarily illustrated in Fig. 4a. The bSSFP signal intensities from two WM and two GM regions from one healthy subject are fitted based on Eq. 11 applying either the constant WM or GM parameters. Resulting MWF values are given in Table 1, together with the sum of squares of the residuals (SSQ). The fitted MWF of the GM regions are considerably smaller than the MWF obtained from WM regions yielding good correspondence with literature values [4,7-14,17,25,36]. WM and GM results from single-pool fitting using the finite RF pulse corrected Freeman-Hill formula are presented in Fig. 4b. For the single-pool fit, the distance between acquired data and Freeman-Hill formula was minimized with respect to the transverse relaxation $T_{2}$ and the signal amplitude, while leaving $T_{1}$ fixed $(850 \mathrm{~ms}$ for WM, 1,200 ms for GM). Due to the dependence on the ratio $T_{2} / T_{1}$ of the Freeman-Hill formula [20], fitting of both relaxation times was not feasible. The resulting single-pool $T_{2}$ values are shown in Table 1, together with the SSQs. In general, both fit procedures yield small residuals of less than $2 \%$. To evaluate the significance, direct comparison of the different SSQs is valid, because both the two-pool and the single-pool fit procedure possess 6 degrees of freedom $(8$ 
Table 2 MWF [\%] derived from pixelwise two-pool bSSFP model fitting of segmented white matter (pixelwise mean \pm pixelwise SD)

\begin{tabular}{|c|c|c|c|c|c|}
\hline MWF [\%] & Subject 1 & Subject 2 & Subject 3 & Subject 4 & Subject 5 \\
\hline Axial view & $11.2 \pm 4.0$ & $13.6 \pm 5.1$ & $14.8 \pm 6.1$ & $12.5 \pm 4.9$ & $11.9 \pm 4.6$ \\
\hline Sagittal view & $12.3 \pm 3.8$ & $13.1 \pm 4.5$ & $14.8 \pm 5.0$ & $12.2 \pm 3.7$ & $11.4 \pm 3.8$ \\
\hline Coronal view & $11.9 \pm 3.3$ & $12.3 \pm 4.0$ & $14.2 \pm 4.7$ & $13.2 \pm 4.4$ & $13.2 \pm 4.2$ \\
\hline
\end{tabular}

Corresponding MWF parameter maps are illustrated in Fig. 5

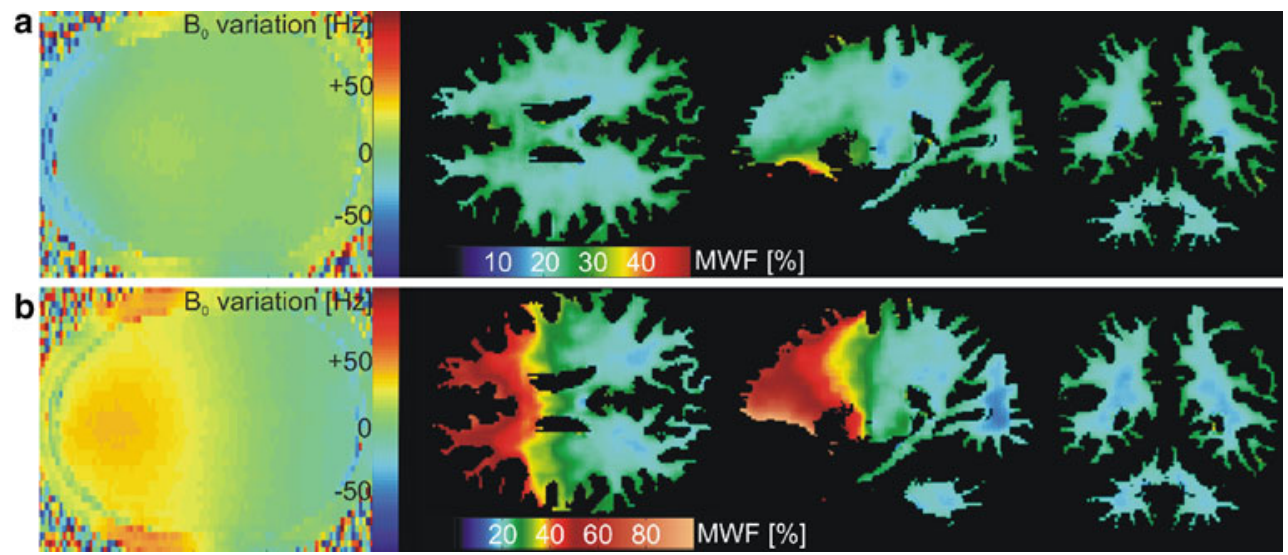

Fig. 6 Right: axial, sagittal and coronal sample images of a sixth healthy volunteer showing $1.3 \mathrm{~mm}$ isotropic high-resolution MWF parameter estimates as derived from two-pool bSSFP model fitting using the NLLS algorithm and the extended bSSFP fit equation (Eq. 11). Left: axial quantitative $B_{0}$ maps having the same location as the axial sample image shown on the right side. Maps were computed based on two gradient recalled echo images with different echo times.

data points-2 unknowns). Since the SSQs from the twopool bSSFP fitting are in each region reduced compared to the singe-pool fitting SSQs, one can conclude that the extended two-pool bSSFP equation yields significantly better fit results than the application of the Freeman-Hill formula.

Axial, sagittal and coronal quantitative myelin water maps with an isotropic resolution of $1.3 \mathrm{~mm}$ are presented in Fig. 5 for WM of five normal appearing human brains. Mean MWF values from segmented WM pixels with corresponding standard deviations are listed in Table 2, separately indicated for the investigated subjects. Besides some small problems with off-resonance artifacts affecting particularly the regions near the nasal cavities, the derived MWF maps appear smooth and high correlation between individual subjects is observed. Furthermore, the mean MWF values correspond to literature values $[4,7-14,17,25,36]$.

To investigate the influence of $B_{0}$ inhomogeneities on the experimental bSSFP data, the measurement protocol of the sixth healthy volunteer was expanded. In addition to the regular protocol described in Section Materials and Methods, a standard Siemens protocol based on two gradient recalled echo sequences with different TE was measured to calculate quantitative $B_{0}$ maps. The field mapping sequence had sagittal orientation and consisted of a $128 \times 128$ matrix with a Regular measurement protocol (as described in Section "Materials and methods"). b Manually induced increase of the $B_{0}$ inhomogeneity by $10 \mu \mathrm{T} / \mathrm{m}$ (in $y$-direction from frontal to posterior) results in frequency variations of about $40 \mathrm{~Hz}$ in the $B_{0}$ map and leads to the formation of an apparent MWF gradient reaching from frontal to posterior WM having the size of $\sim 60 \%$

$2 \times 2 \mathrm{~mm}^{2}$ resolution and 36 slices with $4 \mathrm{~mm}$ slice thickness. $\mathrm{TR}$ was $455 \mathrm{~ms}, \mathrm{TE}_{1}=4.76 \mathrm{~ms}, \mathrm{TE}_{2}=9.52 \mathrm{~ms}, \alpha=60^{\circ}$ and bandwidth $=260 \mathrm{~Hz} /$ pixel. Figure $6 \mathrm{a}$ illustrates the axial, sagittal and coronal sample images showing $1.3 \mathrm{~mm}$ isotropic high-resolution MWF parameter estimates and on the left side one quantitative $B_{0}$ map having the same location as the axial MWF map. Figure $6 \mathrm{~b}$ depicts the second part of the measurement, where the $8 \mathrm{bSSFP}$ sequences were repeated with a manual increase of the $B_{0}$ inhomogeneity by $10 \mu \mathrm{T} / \mathrm{m}$ (in $\mathrm{y}$-direction from frontal to posterior). This modification leads to frequency variations of about $40 \mathrm{~Hz}$ in the $B_{0}$ map and results in the formation of an apparent MWF gradient reaching from frontal to posterior WM. Therefore, it can be concluded that the fit procedure introduced in this work reveals to be highly sensitive to $B_{0}$ variations arising i.e. from dental fillings. For that reason, a careful conducted high order manual shim is essential for obtaining smooth MWF parameter maps.

\section{Discussion}

In this work, a new bSSFP signal equation based on a twopool water exchange model was derived that was used as fit 
equation for the quantitative analysis of bSSFP measurement data with varying flip angles. The distance between acquired data and model was minimized with respect to the MWF and the signal amplitude, while keeping the parameters $T_{1}^{s}, T_{1}^{l}, T_{2}^{s}, T_{2}^{l}$ and $k$ fixed. Obtained results (Tables 1 and 2) correspond to previously reported literature values $[4,7-14,17,25,36]$. Moreover, the MWF parameter results from GM regions are considerably smaller than the MWF obtained from WM regions yielding as well agreement with literature. Aside from that, $T_{1}$ of myelin water was assumed to be considerably shorter than the longitudinal relaxation of the intra- and extracellular water of the axon. Omitting this difference would lead to a serious overestimation of the MWF of about $10 \%$ and therefore result in MWF values inside the upper range of literature values.

On-resonance MT effects were excluded from experiments by choosing long RF pulse durations (Fig. 3b). However, the selection of long RF pulse durations requires the use of a finite pulse correction (Fig. 2), which was applied to the derived bSSFP signal equation. Another important error source is $B_{1}$ field inhomogeneity leading to flip angle deviations of maximal $10 \%$ in the experimental data. To eliminate these systematic errors, the applied flip angles were corrected prior to data analysis, by performing $B_{1}$ field mapping. Furthermore, problems with $B_{0}$ inhomogeneities were avoided by carefully conducting high-order manual shims. The neglect of manual shimming leads to the formation of high MWF gradients in the parameter maps making this sort of correction essential (Fig. 6b). Issues with $B_{0}$ inhomogeneities would as well play an important role in case of switching to higher magnetic field strengths. There, not only problems with MWF gradients would arise, but one would also have to deal with formation of bSSFP off-resonance bands in the acquired images. Nonetheless, by applying careful manual shimming and RF phase cycling to avoid banding artifacts, measurements at higher field strengths may be feasible. Increased imaging time due to the phase cycling approach might be reduced by parallel imaging techniques.

The proposed two-pool analysis procedure is based on fitting the MWF and the signal amplitude and fixing the parameters $T_{2}^{s}, T_{2}^{l}, T_{1}^{s}, T_{1}^{l}$ and $k$. In principle, the choice to fix certain parameters was made based on two reasons. First, the derived bSSFP signal equation is extremely complex making it impossible to fit all parameters, especially in the presence of the simple bSSFP curve behavior. Second, there are four unknown relaxation times present in the twopool model, which cannot be measured directly with other techniques and then inserted backwards into the model. In particular, the knowledge about the two longitudinal relaxation times is very limited from literature. However, it is clear from previous work that the relaxation times show spatial and pathological variation and are not globally constant. To investigate the stability of the MWF parameter, variation of
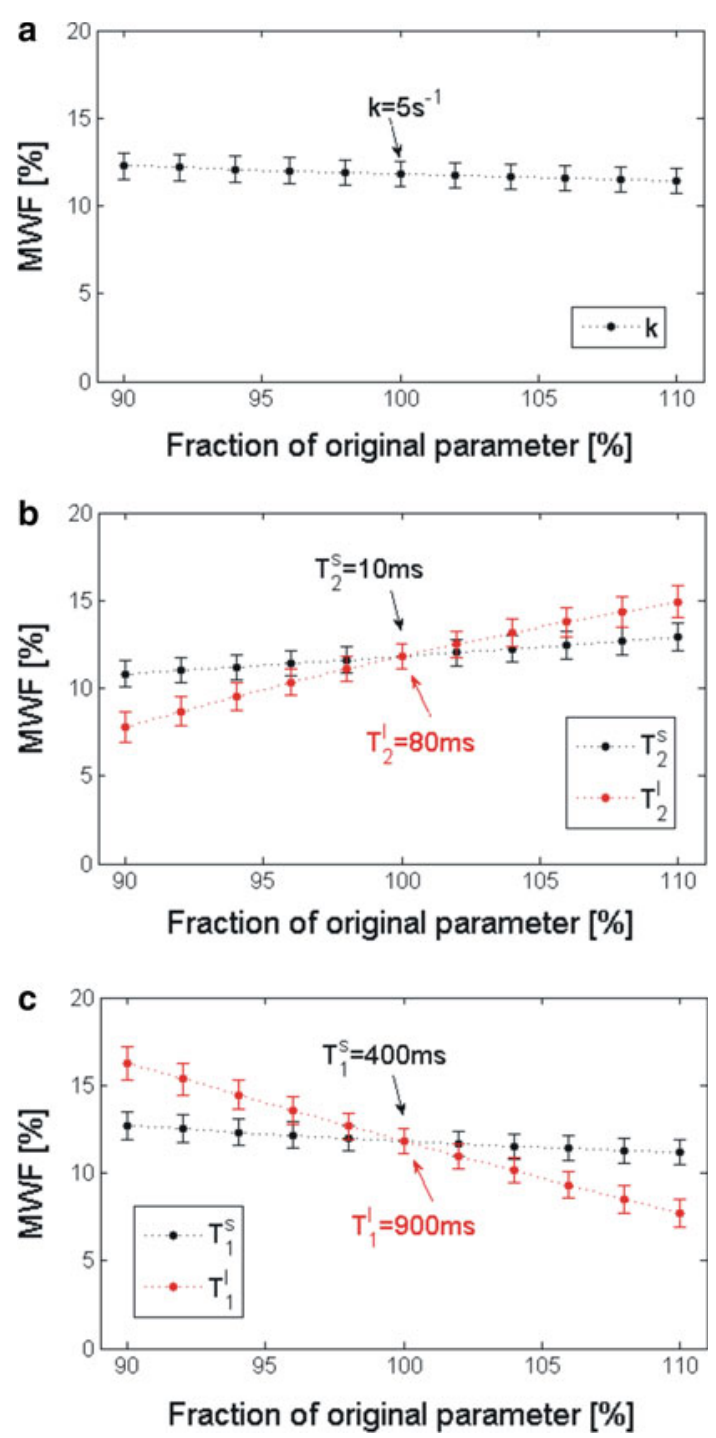

Fig. 7 Estimation of MWF stability upon variation $( \pm 10 \%)$ of constant fit parameters for one WM region. MWF fit results \pm standard errors are given as a function of the parameter variation. In each case, one parameter is varied while leaving the remaining parameters unmodified. a Variation of the exchange rate $k$. b Variation of the transverse relaxation times $T_{2}^{s}$ and $T_{2}^{l}$. $\mathbf{c}$ Variation of the longitudinal relaxation components $T_{1}^{s}$ and $T_{1}^{l}$

the fixed parameters was performed, which is shown in Fig. 7 for one WM region. Thereby, each of the constant parameters was varied between $\pm 10 \%$. Variation of the exchange rate results in extremely small deviation of the fitted MWF $(< \pm 4 \%)$ (Fig. 7a). The discrepancy of the MWF values upon variation of $T_{2}^{s}$ and $T_{1}^{s}$ is as well in a reasonable range $(< \pm 10 \%)$ (Fig. 7b, c). On the other hand, the alteration of the fitted MWF caused by variation of $T_{2}^{l}$ and $T_{1}^{l}$ is more severe. However, the smoothness of the derived MWF parameter maps (Fig. 5) indicates that the "real" spatial WM parameter variation of the investigated healthy subjects must be smaller than $\pm 10 \%$, because the standard deviations of our 


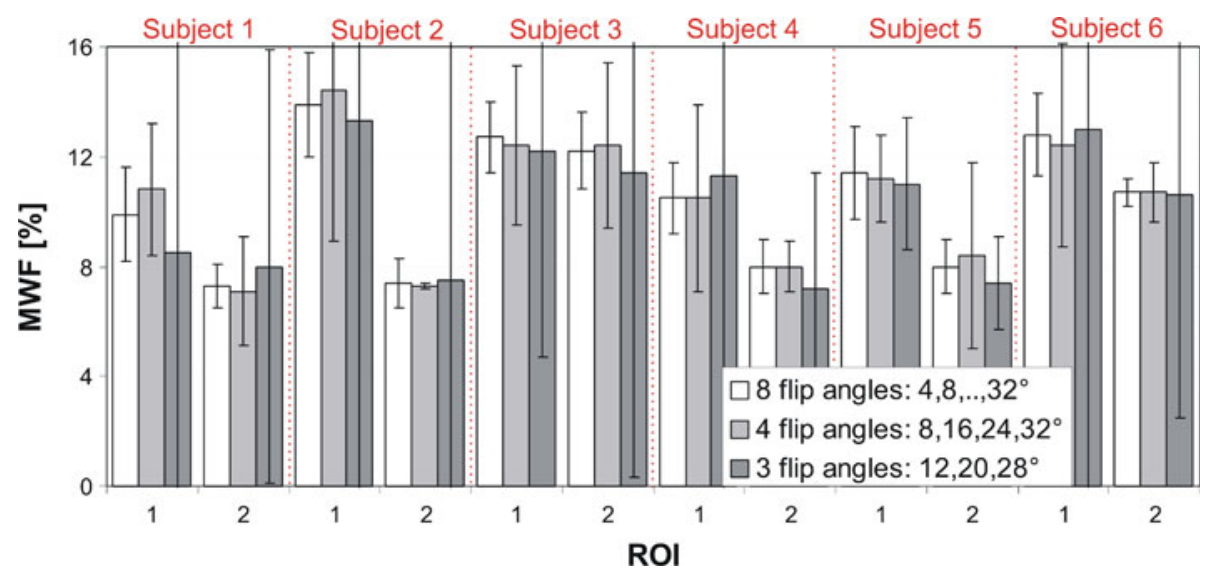

Fig. 8 Fit results of two WM regions evaluated in all healthy subjects showing on the one hand the incorporation of all 8 flip angles to the fit with $\alpha=4^{\circ}, 8^{\circ}, \ldots, 32^{\circ}$ and on the other hand the reduction to 4 flip angles with $\alpha=4^{\circ}, 16^{\circ}, 24^{\circ}, 32^{\circ}$. The proposed flip angle decrease results in similar values for the MWF and slightly enhanced standard errors, which may lead to a possible shortening of measurement time from 25 to $15 \mathrm{~min}$. Due to the extreme high standard errors, the reduction to 3 flip angles is impractical
MWF results are broadly comparable to literature results $[4,7-14,17,25,36]$. Nevertheless, the strong dependence of the MWF on $T_{2}^{l}$ and $T_{1}^{l}$ demonstrates that the applicability of the proposed method not only to pathologies, but also to maturation or ageing of WM is considerably impaired.

Only recently, Deoni et al. introduced a similar approach to myelin water imaging, but used, in contrary to our study, a genetic algorithm to fit all unknown parameters [14]. In their work, presented MWF results from WM pixels of healthy subjects lie inside the upper range of literature values (averaged $\mathrm{MWF}=29.5 \pm 5.3 \%$ ) and are considerably higher than the results presented here. We strongly assume that the observed difference arises not only due to the fixed parameters in our study, but also due to the negligence of $B_{1}$ inhomogeneities and MT effects in the work of Deoni et al.

Lately, some groups predicted the two-pool model to be inappropriate and supposed instead a three pool model including the axonal water as separate pool (fractional pool size $\sim 50 \%)$, the myelin water $(\sim 30 \%)$ and the extracellular water as third pool $(\sim 30 \%)[13,37,38]$. According to these studies, a significant amount of signal from the axonal water could be misclassified into the myelin water signal if only two pools were considered. This, in turn, would lead to an eventual global overestimation of the fitted MWF.

Figure 8 illustrates fit results from WM regions showing on the one hand the incorporation of all 8 flip angles to data analysis and on the other hand the reduction to either 3 or 4 flip angles. Application of 4 different flip angles results in similar values for the MWF and slightly enhanced standard errors, whereas the decrease to 3 flip angles is impractical due to extreme high standard errors $(>100 \%)$. The reduction to 4 flip angles would lead to a considerable shortening of acquisition time from 25 to $15 \mathrm{~min}$.

The technique presented in this work offers a stable approach to high-resolution 3D myelin water imaging of WM from healthy subjects. However, the application of the proposed technique is limited due to the fixed parameters included in the analysis procedure. Nevertheless, we believe that this study provides a non-negligible contribution to the relatively new field of 3D myelin water quantification with whole-brain coverage.

\section{Conclusion}

In this work, a new bSSFP signal equation based on a twopool water exchange model was derived to quantify the MWF of WM from healthy subjects. In the course of the proposed two-pool model analysis, the MWF and the signal amplitude were fitted as single unknowns, while a priori assumptions were made for the other parameters of the two-pool system. The influence of $B_{0}$ and $B_{1}$ inhomogeneities, as well as MT effects and the requirement for a finite RF pulse correction were discussed. Moreover, the stability of the derived MWF values upon variation of the fixed parameters was investigated. MWF values found in this study showed good correspondence with literature. However, the strong dependence of the MWF on the fixed two-pool parameters considerably impairs the application of the proposed technique.

Acknowledgements This work was partially supported by Bayer Schering Pharma Switzerland. 


\section{Appendix}

The extended bSSFP signal equation is written as:

$S \propto \sin \alpha \frac{A+B \cos \alpha}{C+D \cos \alpha+E \cos 2 \alpha}$,

with full abbreviations given by:

$$
\begin{aligned}
& A=e^{2 k T R}\left(1+E_{1}^{l}(\mathrm{MWF}-1)-E_{1}^{s} \mathrm{MWF}\right) \\
& \left(\sqrt{E_{2}^{l}}(\mathrm{MWF}-1)-\sqrt{E_{2}^{s}} \mathrm{MWF}\right) \\
& -e^{k T R}\left(E_{1}^{s}-E_{1}^{l}\right)\left(\sqrt{E_{2}^{s}}+\left(E_{2}^{s}-1\right) \sqrt{E_{2}^{l}}-\sqrt{E_{2}^{s}} E_{2}^{l}\right) \\
& \text { (MWF - 1) MWF } \\
& +\sqrt{E_{2}^{s}} \sqrt{E_{2}^{l}}\left(\sqrt{E_{2}^{s}}(\mathrm{MWF}-1)-\sqrt{E_{2}^{l}} \mathrm{MWF}\right) \\
& \left(-E_{1}^{l} \mathrm{MWF}+E_{1}^{s}\left(-1+E_{1}^{l}+\mathrm{MWF}\right)\right) \\
& B=e^{k T R}\left[\begin{array}{l}
\left(1-E_{1}^{l}\right)\left(E_{1}^{s}-E_{2}^{s}\right) \sqrt{E_{2}^{l}} \ldots \\
+\left(\left(1-E_{1}^{s}\right) E_{1}^{l} \sqrt{E_{2}^{s}}+\left(E_{1}^{l}-1\right)\left(E_{1}^{s}-E_{2}^{s}\right)\right. \\
\left.\times \sqrt{E_{2}^{l}}+\left(E_{1}^{s}-1\right) \sqrt{E_{2}^{s}} E_{2}^{l}\right) \mathrm{MWF}
\end{array}\right] \\
& C=e^{2 k T R}\left[-1+\left(E_{1}^{l}(\mathrm{MWF}-1)-E_{1}^{s} \mathrm{MWF}\right)\right. \\
& \left.\left(E_{2}^{l}(\mathrm{MWF}-1)-E_{2}^{s} \mathrm{MWF}\right)\right] \\
& +\frac{1}{2} e^{k T R}\left[\begin{array}{l}
-\left(E_{1}^{s}-E_{2}^{s}\right)\left(E_{1}^{l}-E_{2}^{l}\right) \\
+4\left(E_{1}^{s}-E_{1}^{l}\right)\left(E_{2}^{s}-E_{2}^{l}\right) \mathrm{MWF}_{2} \ldots \\
-4\left(E_{1}^{s}-E_{1}^{l}\right)\left(E_{2}^{s}-E_{2}^{l}\right) \mathrm{MWF}^{2}
\end{array}\right] \\
& +E_{1}^{l} \mathrm{MWF}\left(E_{2}^{s}(1-\mathrm{MWF})+E_{2}^{l} \mathrm{MWF}\right) \\
& +E_{1}^{s}\left(E_{2}^{s}\left(-E_{1}^{l} E_{2}^{l}+(\mathrm{MWF}-1)^{2}\right)\right. \\
& \left.-E_{2}^{l}(\mathrm{MWF}-1) \mathrm{MWF}\right) \\
& D=e^{2 k T R}\left[E_{1}^{l}-E_{2}^{l}+\left(E_{1}^{s}-E_{1}^{l}-E_{2}^{s}+E_{2}^{l}\right) \mathrm{MWF}\right] \\
& -e^{k T R}\left[\left(E_{1}^{s}-E_{2}^{s}\right)\left(-1+E_{1}^{l} E_{2}^{l}\right)\right. \\
& \left.+\left(\begin{array}{l}
-E_{1}^{l}-E_{2}^{s}+E_{2}^{l}+E_{1}^{l} E_{2}^{s} E_{2}^{l} \ldots \\
+E_{1}^{s}\left(1+E_{1}^{l} E_{2}^{s}-\left(E_{1}^{l}+E_{2}^{s}\right) E_{2}^{l}\right)
\end{array}\right) \mathrm{MWF}\right] \\
& +E_{1}^{l} E_{2}^{s} E_{2}^{l} \mathrm{MWF}-E_{1}^{s}\left(E_{2}^{s} E_{2}^{l}(\mathrm{MWF}-1)\right. \\
& \left.+E_{1}^{l}\left(E_{2}^{s}-E_{2}^{s} \mathrm{MWF}+E_{2}^{l} \mathrm{MWF}\right)\right) \\
& E=-\frac{1}{2} e^{k T R}\left(E_{1}^{s}-E_{2}^{s}\right)\left(E_{1}^{l}-E_{2}^{l}\right)
\end{aligned}
$$

\section{References}

1. Vasilescu V, Katona E, Simplâceanu V, Demco D (1978) Water compartments in the myelinated nerve. III. Pulsed NMR result. Cell Mol Life Sci 34:1443-1444
2. Menon RS, Allen PS (1991) Application of continuous relaxation time distributions to the fitting of data from model systems and excised tissue. Magn Reson Med 20:214-227

3. Stewart WA, MacKay AL, Whittall KP, Moore GRW, Paty DW (1993) Spin-spin relaxation in experimental allergic Encephalomyelitis. Analysis of CPMG data using a non-linear least squares method and linear inverse theory. Magn Reson Med 29:767-775

4. MacKay AL, Whittall KP, Adler J, Li DKB, Paty DW, Graeb DA (1994) In vivo visualization of myelin water in brain by magnetic resonance. Magn Reson Imaging 31:673-677

5. Lawson CL, Hanson RJ (1974) Solving least squares problems. Prentice-Hall, Englewood Cliffs

6. Whittall KP, MacKay AL (1989) Quantitative interpretation of NMR relaxation data. J Magn Reson 84:134-152

7. Laule C, Leung E, Li DK, Traboulsee AL, Paty DW, MacKay AL, Moore GR (2006) Myelin water imaging in multiple sclerosis: quantitative correlations with histopathology. Mult Scler 12: 747-753

8. MacKay AL, Laule C, Vavasour IM, Bjarnason TA, Kolind S, Madler B (2006) Insights into brain microstructure from the $T_{2}$ distribution. Magn Reson Imaging 24:515-525

9. Laule C, Kozlowski P, Leung E, Li DKB, MacKay AL, Moore GRW (2008) Myelin water imaging of multiple sclerosis at $7 \mathrm{~T}$ : correlations with histopathology. Neuroimage 40:1575-1580

10. Flynn SW, Lang DJ, MacKay AL, Goghari V, Vavasour IM, Whittall KP, Smith GN, Arango V, Mann JJ, Dwork AJ, Falkai $\mathrm{P}$, Honer WG (2003) Abnormalities of myelination in schizophrenia detected in vivo with MRI, and post-mortem with analysis of oligodendrocyte proteins. Mol Psychiatry 8:811-820

11. Sirrs SM, Laule C, Madler B, Brief EE, Tahir SA, Bishop C, MacKay AL (2007) Normal-appearing white matter in patients with phenylketonuria: water content, myelin water fraction, and metabolite concentrations. Radiology 242:236-243

12. Oh J, Han ET, Pelletier D, Nelson SJ (2006) Measurement of in vivo multi-component $T_{2}$ relaxation times for brain tissue using multi-slice $\mathrm{T}_{2}$ prep at 1.5 and $3 \mathrm{~T}$. Magn Reson Imaging 24:33-43

13. Du YP, Chu R, Hwang D, Brown MS, Kleinschmidt-DeMasters BK, Singel D, Simon JH (2007) Fast multislice mapping of the myelin water fraction using multicompartment analysis of $\mathrm{T}_{2} *$ decay at 3T: A preliminary postmortem study. Magn Reson Med $58: 865-870$

14. Deoni SCL, Rutt BK, Arun T, Pierpaoli C, Jones DK (2008) Gleaning multicomponent $\mathrm{T}_{1}$ and $\mathrm{T}_{2}$ information from steady-state imaging data. Magn Reson Med 60:1372-1387

15. Does MD, Beaulieu C, Allen PS, Snyder RE (1998) Multicomponent $\mathrm{T}_{1}$ relaxation and magnetisation transfer in peripheral nerve. Magn Reson Imaging 16:1033-1041

16. Bjarnason TA, Vavasour IM, Chia CLL, MacKay AL (2005) Characterization of the NMR behavior of white matter in bovine brain. Magn Reson Med 54:1072-1081

17. Stanisz GJ, Kecojevic A, Bronskill MJ, Henkelman RM (1999) Characterizing white matter with magnetization transfer and $\mathrm{T}_{2}$. Magn Reson Med 42:1128-1136

18. Oppelt A, Graumann R, Barfuss H, Fischer H, Hartl W, Schajor W (1986) FISP: eine neue schnelle Pulssequenz für die Kernspintomographie. Electromedia 54:15-18

19. Gloor M, Scheffler K, Bieri O (2008) Quantitative magnetization transfer imaging using balanced SSFP. Magn Reson Med 60: 691-700

20. Scheffler K, Lehnhardt S (2003) Principles and applications of balanced SSFP techniques. Eur Radiol 13:2409-2418

21. Freeman R, Hill HDW (1971) Phase and intensity anomalies in fourier transform NMR. J Magn Reson 4:366-383

22. Zur Y, Stokar S, Bendel P (1988) An analysis of fast imaging sequences with steady-state transverse magnetization refocusing. Magn Reson Med 6:175-193 
23. Deoni SCL, Rutt BK, Jones DK (2008) Investigating exchange and multicomponent relaxation in fully-balanced steady-state free precession imaging. J Magn Reson Imaging 27:1421-1429

24. Koenig SH, Brown RDIII, Spiller M, Lundbom N (1990) Relaxometry of brain: why white matter appears bright in MRI. Magn Reson Med 14:482-495

25. Vavasour IM, Whittall KP, Li DKB, MacKay AL (2000) Different magnetization transfer effects exhibited by the short and long $\mathrm{T}_{2}$ components in human brain. Magn Reson Med 44:860-866

26. Bloch F (1946) Nuclear induction. Phys Rev 70:460-474

27. Carr HY (1958) Steady-state free precession in nuclear magnetic resonance. Phys Rev 112:1693-1701

28. Jaynes ET (1955) Matrix treatment of nuclear induction. Phys Rev 98:1099-1105

29. Haacke EM, Brown RW, Thompson MR, Venkatesan R (1999) Magnetic resonance imaging: physical principles and sequence design. John Wiley, New York

30. Bieri O, Scheffler K (2006) On the origin of apparent low tissue signals in balanced SSFP. Magn Reson Med 56:1067-1074

31. Bieri O, Scheffler K (2007) Optimized balanced steady-state free precession magnetization transfer imaging. Magn Reson Med 58:511-518

32. Akoka S, Franconi F, Seguin F, Le Pape A (1993) Radiofrequency map of an NMR coil by imaging. Magn Reson Imaging 11:437-441
33. Smith SM, Jenkinson M, Woolrich MW, Beckmann CF, Behrens TEJ, Johansen-Berg H, Bannister PR, De Luca M, Drobnjak I, Flitney DE, Niazy RK, Saunders J, Vickers J, Zhang Y, De Stefano N, Brady JM, Matthews PM (2004) Advances in functional and structural MR image analysis and implementation as FSL. Neuroimage 23:208-219

34. Cox RW (1996) AFNI: software for analysis and visualization of functional magnetic resonance neuroimages. Comput Biomed Res 29:162-173

35. Bieri O, Scheffler K (2009) The impact of finite RF excitation on steady state free precession. In: Proceedings of seventeenth scientific meeting, International Society for Magnetic Resonance in Medicine 2793

36. Whittall KP, MacKay AL, Graeb DA, Nugent RA, Li DKB, Paty DW (1997) In vivo measurement of $T_{2}$ distributions and water contents in normal human brain. Magn Reson Med 37:34-43

37. Lancaster JL, Andrews T, Hardies LJ, Dodd S, Fox PT (2003) Three-pool model of white matter. J Magn Reson Imaging 17: $1-10$

38. Trevor A, Lancaster JL, Dodd SJ, Contreras-Sesvold C, Fox PT (2005) Testing the three-pool white matter model adapted for use with $\mathrm{T}_{2}$ relaxometry. Magn Reson Med 54:449-454 